Pneumologe 2018 $\cdot 15: 263-265$

https://doi.org/10.1007/s10405-018-0192-8

(c) Springer Medizin Verlag GmbH, ein Teil von Springer Nature 2018

\section{Redaktion}

R. Loddenkemper, Berlin

H. Olschewski, Graz

CrossMark

Der Dieselskandal hat eine heftige, emotional geführte Debatte über die gesundheitlichen Folgen der Exposition mit Kfzverkehrsabhängigen Schadstoffen insbesondere in Deutschland ausgelöst. Diese Debatte ist überwiegend in den Medien geführt worden und erweckte mitunter intendiert oder unbeabsichtigt den Eindruck, als sei sich die Wissenschaft über die gesundheitlichen Folgen dieser Schadstoffexpositionen uneins. Deswegen ist eine sachliche Klarstellung zur Evidenz für die nachteiligen Wirkungen dieser Luftschadstoffe erforderlich. Die Deutsche Gesellschaft für Pneumologie und Beatmungsmedizin (DGP) sieht sich als eine der zuständigen medizinischen Fachgesellschaften diesem Anliegen verpflichtet und hat deswegen dieses Thema anlässlich ihres Jahreskongresses aufgegriffen, um Stellung zu beziehen.

Die provokant aufgeworfene Frage kann man kurz und eindeutig mit einem klaren "Ja“ beantworten. Die lufthygienische Situation hat sich in Deutschland während der letzten Jahrzehnte deutlich verbessert. Dennoch gibt es ausreichende Gründe, eine weitere Verbesserung einzufordern und entsprechende Maßnahmen einzuleiten. Im Nachfolgenden werden ein Überblick über die aktuelle Datenlage zu Wirkungen von verkehrsabhängigen Luftschadstoffexpositionen auf Lunge und Atemwege gegeben und

Dieser Beitrag ist im Rahmen der Pressekonferenz zum 59. Kongresses der Deutschen Gesellschaft für Pneumologie und Beatmungsmedizin (DGP), Mittwoch, 7. März 2018, Tagungszentrum im Haus der Bundespressekonferenz, Berlin bereits veröffentlicht worden (Abdruck mit freundlicherGenehmigung derDGP).

\author{
J. Heinrich ${ }^{1,2}$ \\ ${ }^{1}$ Institut und Poliklinik für Arbeits-, Sozial- und Umweltmedizin, WHO, Collaborating Centre for \\ Occupational Health, Klinikum der Universität München, München, Deutschland \\ ${ }^{2}$ Deutsches Zentrum für Lungenforschung, Gießen, Deutschland
}

\title{
Feinstaub, Stickoxide, Ozon: Darf man in Großstädten noch atmen?
}

Maßnahmen zur weiteren Verbesserung diskutiert.

\section{Quellen der Luftverschmutzung in unseren Städten}

Es gibt im Wesentlichen folgende Quellen, die die Luftverschmutzung in unseren Städten beeinflussen: die Emissionen des lokalen Straßenverkehrs sowie der Ferntransport von Luftschadstoffen, die der Industrie oder Landwirtschaft entstammen. Hinzu kommt in manchen Gegenden die Emission aus Kleinfeuerungsanlagen, insbesondere bei Verbrennung von Holz oder Holzpellets. Zu den Emissionen des Autoverkehrs gehören neben den Abgasen auch der nicht zu vernachlässigende Abrieb von Bremsen und Reifen sowie die Wiederaufwirbelung von sedimentiertem Staub. Die Emissionen des Autoverkehrs und dabei der Dieselfahrzeuge insbesondere tragen maßgeblich zur Luftbelastung mit Stickoxiden $\left(\mathrm{NO}_{\mathrm{x}}\right)$ bei. Zusätzlich sind die älteren Dieselfahrzeuge ohne Partikelfilter eine maßgebliche Quelle der Feinstaubbelastung, messbar als Partikel mit einem aerodynamischen Durchmesser von kleiner als $10 \mu \mathrm{m}\left(\mathrm{PM}_{10}\right)$ sowie als Partikel mit einem Durchmesser von kleiner als 2,5 $\mu \mathrm{m}$ $\left(\mathrm{PM}_{2,5}\right)$. Feinstaub und Stickoxide, insbesondere Stickstoffdioxid $\left(\mathrm{NO}_{2}\right)$, sind neben Ozon in bodennahen Luftschichten die Luftschadstoffe, deren gesundheitliche Wirkung derzeit am besten untersucht ist und die wegen ihrer Schadwirkung in landesweiten Messnetzen kontinuierlich gemessen werden. Der Gesetzgeber hat Grenzwerte zum Schutz der Bevölkerung vor gesundheitlichen Gefahren festgeschrieben sowie Maßnahmen zur Reduktion der Schadstoffkonzentration empfohlen.

\section{Worin bestehen hauptsächlich gesundheitliche Gefahren durch Luftverschmutzung für die Lunge und die Atemwege?}

Die nachteiligen gesundheitlichen Wirkungen von Luftschadstoffen sind durch experimentelle Expositionsstudien, durch Human-Expositionsstudien und schließlich durch epidemiologische Studien ausreichend belegt (s. Übersichtsartikel $\mathrm{zu}$ Luftschadstoffen und Lunge von Perez et al. [15]). Während die gesundheitlichen Wirkungen im Zusammenhang mit einzelnen Schadstoffen wie Feinstaub, Stickstoffdioxid und Ozon ausreichend erwiesen sind, ist der spezielle $\mathrm{Zu}$ sammenhang mit den Emissionen des Autoverkehrslediglich in Form eines ,Indiziennachweises" zu erbringen, weil die eingeatmete Luft stets aus einem Gemisch verschiedener Schadstoffe unterschiedlicher Quellen besteht. Als „Indizien“ für die gesundheitlichen Schadwirkungen des Autoverkehrs dienen der gesicherte Nachweis, dass Autoabgase zu den Luftschadstoffkonzentrationen in unseren Städten beitragen, die zahlreichen Belege, dass Wohnen nahe verkehrsreichen Straßen mit höheren Erkrankungsrisiken einhergeht (auch nach dem Herausrechnen möglicher Straßenlärmeffekte sowie unter Berücksichtigung weiterer Faktoren wie die unterschiedliche Sozialstruktur in Abhängigkeit von der Straßennähe) und dass bestimmte Komponenten im Feinstaub, die spezifisch für die Kraftfahrzeugabgase sind, mit gesundheitlich erhöhten Risiken assozi- 
iert sind. Der epidemiologische Nachweis von irreversiblen gesundheitlichen Langzeitwirkungen entstammt bisher überwiegend den Ergebnissen umfangreicher Kohortenstudien in Nordamerika, die teils über Jahrzehnte hinweg auch im Hinblick auf die Entstehung von Atemwegserkrankungen untersucht wurden. Die Ergebnisse dieser Studien begründeten maßgeblich die derzeitigen Grenzwerte für $\mathrm{PM}_{10}$-Feinstaub und Stickoxide in Europa. Dabei mussten vielfältige Unterschiede zwischen Nordamerika und Europa in Kauf genommen werden: $z$. B. Unterschiede in der Emittentenstruktur, der Luftchemie wegen anderer klimatischer Bedingungen. Deshalb sind zusätzlich Daten zu Langzeitwirkungen von Luftschadstoffen in Europa erforderlich, um auch hier die Allgemeinbevölkerung ausreichend vor diesen Schadwirkungen zu schützen. Die Etablierung und Langzeitbeobachtung ausreichend großer Kohorten in Europa ist aber extrem teuer, aufwendig und dauert zu lange, um jetzt entsprechende Novellierungen der Grenzwertfestsetzung zu begründen. Genau an diesem Punkt setzt das europäische ESCAPEProjekt an, um Abhilfe bei Wissenslücken zu schaffen.

ESCAPE (European Study of Cohorts for Air Pollution Effects) ist ein europäisches Forschungsprojekt zur Quantifizierung von Langzeitwirkungen von Luftschadstoffen wie $\mathrm{PM}_{10^{-}}$und $\mathrm{PM}_{2,5^{-}}$ Feinstaub, Feinstaubinhaltsstoffen wie Metallen, Stickoxiden und Dieselruß auf die menschliche Gesundheit (www. escapeproject.eu). Dabei wird auf existierende Daten von 22 Kohorten zurückgegriffen. Aus Deutschland fließen mit Bezug auf Atemwegserkrankungen Ergebnisse der Erwachsenenkohorten KORA aus der Region Augsburg und SALIA aus Nordrhein-Westfalen sowie der Geburtskohorten GINIplus und LISAplus aus den Regionen München, dem Rheinland und Leipzig ein. Die Besonderheit von ESCAPE liegt allerdings in der standardisierten Modellierung der individuellen Schätzung der Exposition mit Feinstaub und Stickoxiden [3, 6] mittels „land use regression (LUR) models“. Dabei wird ein eigens für ESCAPE entwickeltes Messprogramm an 20-40 Mess- punkten pro Kohorte benutzt, um die Vorhersage der Schadstoffkonzentrationen durch Daten des geografischen Informationssystems (GIS) zu modellieren. Diese Regressionsmodelle werden zur Schätzung der Schadstoffkonzentration an den Wohnadressen der Probanden herangezogen.

\section{Aktuelle, ausgewählte Ergebnis- se zu Atemwegserkrankungen}

Die Analyse der Längsschnittdaten von über 300.000 Probanden aus 17 Kohorten zeigte nach einer Follow-up-Zeit von durchschnittlich 12,8 Jahren ein um $22 \%$ statistisch signifikant erhöhtes Risiko für Lungenkrebs für $\mathrm{PM}_{10}$, allerdings keine Risikoerhöhung für $\mathrm{NO}_{2}$ [16]. Ein jüngst publiziertes systematisches Review zur $\mathrm{NO}_{2}$-Exposition und $\mathrm{zu}$ Lungenkrebs zeigte allerdings ein konsistent erhöhtes Risiko [8]. Die Mortalität an nichtmalignen respiratorischen Ursachen war bei den ESCAPEKohorten nicht statistisch signifikant erhöht [5]. Die Prävalenz und Inzidenz einer chronisch-obstruktiven Lungenerkrankung (COPD) in Abhängigkeit von der Schadstoffexposition wurde bei 6550 Probanden aus 4 multizentrischen Kohorten (ECRHS, SALIA, SAPALDIA, NSHD) untersucht. Es zeigten sich positive, aber nicht statistisch signifikante Risikoerhöhungen [17]. Lediglich für Frauen und teils für Nie-Raucher waren die Risiken teils statistisch signifikant erhöht. Bei Hinzuziehung von Daten der französischen EGEA-Studie zeigten sich in den insgesamt 5 Kohorten statistisch signifikant niedrigere Lungenfunktionswerte $\left(\mathrm{FEV}_{1}, \mathrm{FVC}\right)$ in Abhängigkeit von $\mathrm{PM}_{10}$ und $\mathrm{NO}_{2}$, aber nicht für weitere Schadstoffe. Langzeitexpositionen mit Luftschadstoffen waren nicht assoziiert mit Symptomen der chronischen Bronchitis, Husten oder Auswurf bei den über 10.000 Erwachsenen aus 5 europäischen Kohorten [2]. Allerdings war das Risiko für Auswurf bei Nie-Rauchern mit Bezug auf die „coarse PM“ (PM $\left.\mathrm{PM}_{10-2,5}\right)$ erhöht. Für Kinder waren positive, aber nicht statistisch signifikant erhöhte Risiken für Asthma sowohl in den ESCAPEKohorten [14] als auch in dem jüngst publizierten systematischen Review [9] nachweisbar. Auch für Erwachsene zeigte sich eine leicht erhöhte Asthmainzidenz mit höherer Luftschadstoffexposition, die aber ähnlich wie bei den Kinderstudien nicht als statistisch signifikant $\mathrm{zu}$ sichern war [12]. Die Lungenfunktion $\left(\mathrm{FEV}_{1}, \mathrm{FVC}\right)$ war bei etwa 6000 Kindern aus 5 Kohorten geringfügig, aber statistisch signifikant erniedrigt sowohl für $\mathrm{PM}_{2,5}$ als auch für $\mathrm{NO}_{\mathrm{x}}$, wenn die aktuelle Adresse zur Expositionsschätzung herangezogen wird [7]. Deutliche Effekte für alle modellierten Luftschadstoffexpositionen wurden für Pneumonie nachgewiesen, während keine substanziellen Effekte für das Auftreten von Pseudokrupp beobachtet wurden. Otitis media war statistisch signifikant mit $\mathrm{NO}_{2}$ assoziiert [13].

\section{Welche Bevölkerungsgruppen bedürfen eines besonderen Schutzes?}

Insgesamt sind die Wirkungen von Luftschadstoffen für die Allgemeinbevölkerung in den Konzentrationen, wie wir sie derzeit in Deutschland haben, eindeutig nachweisbar. Obwohl die Stärke der Schadwirkung, der sich niemand entziehen kann, als nicht sehr hoch einzuschätzen ist, betrifft diese dennoch einen sehr großen Anteil unserer Bevölkerung. Deshalb hat der Gesetzgeber auch für saubere Luft zum Atmen für alle, auch für jene, die in Ballungsräumen mit einem hohen Verkehrsaufkommen wohnen, zu sorgen. Ungeachtet dessen gibt es einige Bevölkerungsgruppen, die als besonders verletzlich durch Luftschadstoffe gelten. Dazu gehören Patienten mit geschädigten Atemwegen wie Asthmatiker oder Patienten mit COPD [10], Patienten mit anderen chronischen Erkrankungen wie z. B. Herz-Kreislauf-Erkrankungen, Diabetes sowie Kinder.

\section{Gibt es eine Rechtfertigung, die bestehenden Grenzwerte zu lockern?}

Insgesamt hat sich die Evidenz für die gesundheitliche Schädigung von Lunge und Atemwegen durch Luftschadstoffe wie Feinstaub und Stickoxide und dabei insbesondere für verkehrsabhän- 
gige Luftschadstoffe weiter erhöht. Eine belastbare Bewertung gesundheitlicher Wirkungen darf nicht bei Lunge und Atemwegen als Zielorganen stehen bleiben und muss selbstverständlich HerzKreislauf-Erkrankungen [1], vielleicht auch Diabetes [11], Demenz und kognitive Störungen einbeziehen. Wichtig ist dabei auch, anzumerken, dass gesundheitlich nachteilige Wirkungen auch unterhalb der bestehenden Grenzwerte deutlich zu sehen sind. Es gibt keinen vernünftigen Grund, die bestehenden Grenzwerte für Feinstaub und Stickstoffdioxid infrage zu stellen.

\section{Welche Luftschadstoffe sind besonders schädlich?}

Der Dieselskandal hat die öffentliche Aufmerksamkeit auf die Stickoxide gelenkt. Das hat zu skurrilen Vorschlägen und Gedankenspielen geführt, wie z. B., die Grenzwerte für Feinstaub zu erhöhen, damit durch eine veränderte Technologie der Motorverbrennung der Abgasausstoß von Stickoxiden gesenkt werden kann und die Grenzwerte für Stickoxide somit eingehalten werden könnten (Welt am Sonntag, 28.08.2017). Feinstaub ist aber der nachweislich gesundheitlich schädlichere Luftschadstoff im Vergleich zu den Stickoxiden. Das haben Ergebnisse der ESCAPE-Studie eindrucksvoll bestätigt. Die WHO sieht das genauso wie auch ein jüngst publizierter Übersichtsartikel [20].

Was kann getan werden, um den gesundheitlichen Wirkungen von Feinstaub zu begegnen? Mit einem Bündel von Maßnahmen muss um die Einhaltung dieser Grenzwerte, die schließlich Gesetzescharakter haben, gerungen werden. Dazu gehören im Einzelnen:

- Anforderungen an die Automobilindustrie, durch technologische Maßnahmen den Schadstoffausstoß von Feinstaub und Gasen, insbesondere von Stickstoffoxiden, aus denen sich kleinste Partikel bilden können, zu verringern,

- eine attraktivere Gestaltung des öffentlichen Personennahverkehrs,

- verkehrsorganisatorische Maßnahmen zur Vermeidung des Stop-and-
go-Verkehrs, der mit einem höheren Schadstoffausstoß verbunden ist,

- als ultimative Entscheidung sind auch Fahrverbote in Erwägung zu ziehen,

- die Ausweitung von Umweltzonen als Übergangsmaßnahme, bis andere Maßnahmen zu einer Einhaltung der Grenzwerte geführt haben.

\section{Was kann der Einzelne tun, um die persönliche Schadstoffexposition zu mindern?}

Anzumerken ist dabei zunächst, dass es sich um eine staatliche Aufgabe handelt, dafür Sorge zu tragen, dass die Allgemeinbevölkerung (also auch jene, die in Ballungsräumen leben und die besonders verletzlichen Personengruppen unserer Bevölkerung) saubere Luft einatmen kann. Dennoch kann der Einzelne selbst die Schadstoffexposition reduzieren. So hat eine Studie mit 60 älteren Personen in London, die entlang einer stark befahrenen Straße und davor und danach in dem wenig belasteten Hyde Park gelaufen sind, gezeigt, dass die Lungenfunktion beim Laufen in der weniger verschmutzten Gegend besser wurde [18]. Zusätzlich gab es positive Effekte auf die Herzparameter. Also gibt es gute Gründe, Gegenden, die besonders hoch durch den Straßenverkehr belastet sind, zu meiden, sofern das möglich ist. Sinnvoll ist es auch nach wie vor, die mittlerweile gut etablierten Warnhinweise bei hohen Ozonkonzentrationen (oder Pollenflug) $\mathrm{zu}$ befolgen.

\section{Was haben Maßnahmen zur Feinstaubreduktion bislang gebracht?}

Nachdem die Wirksamkeit von Umweltzonen kurz nach deren Einführung kritisch und bis heute kontrovers diskutiert wurde, mehren sich die Hinweise dafür, dass diese zu einer Reduktion von Schadstoffkonzentrationen führen können, wenn die Umweltzonen ausreichend groß sind (s. Bericht des Umweltbundesamtes [19]; sowie Cyrys et al. [4]). Verkehrsbeschränkungen und zeitweise Fahrverbote führten in verschiedenen Metropolregionen zu einer Abnahme der Luftschadstoffkonzentrationen. Es ist plausibel, anzunehmen, dass durch verkehrsorganisatorische Maßnahmen sowie die Reduktion des Individualverkehrs die Emissionen von Luftschadstoffen verringert werden. Zu guter Letzt steht aber auch die Frage, welche gesundheitlichen Kosten und Nachteile an Lebensqualität durch den Autoverkehr in unserer Wohnumgebung wir bereit sind, in Kauf zu nehmen, im Vergleich zu dem alltäglichen Nutzen unserer privaten Kraftfahrzeugnutzung.

\section{Korrespondenzadresse}

\section{Prof. Dr. J. Heinrich}

Institut und Poliklinik für Arbeits-, Sozial- und Umweltmedizin, WHO, Collaborating Centre for Occupational Health, Klinikum der Universität München

München, Deutschland

Joachim.Heinrich@med.uni-muenchen.de

Interessenkonflikt. J. Heinrich gibt an, dass kein Interessenkonflikt besteht.

\section{Literatur}

1. Brooketal (2010) Circulation 121(21):2331-2378

2. Cai et al (2014) Thorax 69:1005-10149

3. Cyrys et al (2012) Atmos Environ 62:374-390

4. Cyrys et al (2014) J Air Waste Manag Assoc 64(4):481-487

5. Dimakopoulou, I (2014) Am J Respir Crit Care Med 189:684-69610

6. Eeftens etal (2012) Atmos Environ 62:303-317122

7. Gehring et al (2013) Environ Health Perspect 121:1357-1364

8. Hamra et al (2015) Environ Health Perspect 123(11):1107-1112

9. Heinrich et al (2016) Geoinfor Geostat Overv 4:4

10. Heinrich J, Schikowski T (2018) Curr Environ Health $\operatorname{Rep} 5(1): 70-76$

11. Heinrich J, Thiering E (2015) Trends Endocrinol Metab 26(7):384-394

12. Jasquemin et al (2015) Environ Health Perspect 123:613-621

13. Maclntyre et al (2014) Environ Health Perspect 122:107-11325

14. Molter et al (2015) Eur Respir J 45:610-62421

15. Perez Let al (2010) Swiss Med Wkly 140:w13129

16. Raaschou-Nielsen et al (2013) Lancet Oncol 14:813-822230

17. Schikowski et al (2014) Eur Respir J 44:614-62624

18. Sinharay et al (2017) Lancet. https://doi.org/10. 1016/S0140-6736(17)32643-0

19. Umweltbundesamt (2017) www.umweltbundes amt.de/.../analyse-der-wirksamkeit-vonumwelt zonen-in-drei.Zugegriffen: 27.05.2018

20. Wichmann (2018) Umweltmed Hyg Arbeitsmed 23(2):57-71 